\title{
Increased Risk for Falling Associated with Subtle Cognitive Impairment: Secondary Analysis of a Randomized Clinical Trial
}

\author{
Carey E. Gleason ${ }^{\mathrm{a}, \mathrm{c}}$ Ronald E. Gangnon ${ }^{\mathrm{b}}$ Barbara L. Fischer ${ }^{\mathrm{d}}$ Jane E. Mahoney ${ }^{\mathrm{a}}$ \\ aSection of Geriatrics and Gerontology, Department of Medicine, and bepartments of Biostatistics and \\ Medical Informatics and Population Health Sciences, University of Wisconsin, ' ${ }^{C}$ Geriatric Research, Education and \\ Clinical Center (GRECC), and d Mental Health Service, William S. Middleton Memorial Veterans Hospital, \\ Madison, Wisc., USA
}

\section{Key Words}

Accidental falls $\cdot$ Falls $\cdot$ Mini Mental State Exam $\cdot$ Cognition $\cdot$

Geriatrics $\cdot$ Older adults $\cdot$ Risk factors

\begin{abstract}
Background/Aims: Having dementia increases patients' risk for accidental falls. However, it is unknown if having mild cognitive deficits also elevates a person's risk for falls. This study sought to clarify the relationship between subtle cognitive impairment, measured with a widely-used, clinicbased assessment, the Mini Mental State Exam (MMSE), and risk for falls. Methods: In a secondary analysis of the Kenosha County Falls Prevention Study, a randomized controlled trial targeting older adults at risk for falls, we examined the association between baseline MMSE and prospective rate of falls over 12 months in 172 subjects randomized to control group. Results: Using univariate analysis, the rate of falls increased with each unit decrease in MMSE score down to at least 22 (rate ratio 1.25, 95\% confidence interval (CI) 1.09$1.45, p=0.0026)$. Using stepwise multivariate regression, controlling for ability to perform activities of daily living, use of assistive device, current exercise, and arthritis, the asso-
\end{abstract}

ciation between MMSE score and falls rate persisted (rate ratio $1.20,95 \% \mathrm{Cl} 1.03-1.40, p=0.021)$. Conclusion: Minimal decrements on the MMSE were associated with elevations in rate of falls, suggesting that subtle cognitive deficits reflected in MMSE scores above a cut-off consistent with a diagnosis of dementia, can influence risk for falls.

Copyright $\odot 2009$ S. Karger AG, Basel

\section{Introduction}

It has been established that risk of falls increases for community-dwelling patients with dementia [1], and for those with a Mini-Mental State Exam [2] (MMSE) score $\leq 24$ out of 30 [3-5]. Using a standard cut-off score for one of the most widely-used screening instruments, the MMSE [6], investigators found that the predictive relationship between rate of falls and MMSE score was largely driven by individuals scoring in an impaired range $(\leq 24 / 30)$ [3]. These data may give the impression that mild impairment in cognition, not meeting the diagnosis of dementia, or mild decrement in MMSE score, above the cut-off of 24, is not associated with increased risk for

\section{KARGER}

๑ 2009 S. Karger AG, Basel

Fax +4161306 1234 E-Mail karger@karger.ch www.karger.com www.karger.com/dem
Carey E. Gleason, $\mathrm{PhD}$

Section of Geriatrics, Department of Medicine, University of Wisconsin

Madison VA GRECC, Room D4211, 2500 Overlook Terrace

Madison, WI 53705 (USA)

Tel. +1 608256 1901, ext. 17000, Fax +1 608280 7165, E-Mail ceg@medicine.wisc.edu 
falls. In contrast, data from the Kenosha County Fall Prevention Study [7] suggested that mild cognitive deficits may be associated with an increased risk for falls. Specifically, subjects scoring below 28/30 on the MMSE demonstrated a nearly three-fold increased risk for falls compared to control subjects whose MMSE was 30/30. However, the association between MMSE $<28$ and falls was a univariate finding, and it was not clear if the association would remain after controlling for other risk factors of falls. Overall, the relationship between subtle cognitive deficits and rate of falls needs further clarification. We asked the question, 'Does rate of falls increase only after a threshold (such as MMSE less than 24) is reached, or does rate of falls increase incrementally as MMSE score progressively declines?'

Using data from the control group of the Kenosha County Falls Prevention Study, we sought to clarify the relationship between subtle cognitive impairment and risk for falls by examining how subjects' MMSE scores relate to rate of falls. The Kenosha County sample provided the opportunity to examine prospective falls rate in a predominantly non-demented sample. We hypothesized that in a sample of community-dwelling older adults at risk for falls, MMSE scores less than 30 would be associated with increased rate of falls after controlling for other risk factors.

\section{Methods}

Of the full sample included in the Kenosha County Falls Prevention study, 175 older adults were randomized to the control condition and followed for 1 year. Baseline data and prospectively-tracked rate of falls from these individuals were examined in order to clarify the relationship between global cognition and risk of falls. The goals of the original study, the Kenosha County Falls Prevention Study, were to examine the effect of the intervention upon rate of falls, hospitalizations and nursing home placement. Specific details of the study are published elsewhere [7]; a brief description is provided here. The University of Wisconsin (UW) School of Medicine and Public Health Human Subjects Committee approved all procedures, personnel, and analyses associated with the Kenosha County Falls Prevention Study.

\section{Kenosha County Falls Prevention Study}

Subjects. Community-living, older adults at risk for falling were recruited from several locations in Kenosha County, Wisconsin. Specific inclusion criteria were age 65 years or older, living independently in Kenosha County and an elevated risk for falling. Increased risk for falling was defined as (1) a history of 2 or more falls in the last year, or (2) one fall within 2 years prior to entering the study with either injury or gait and balance problems. Exclusion criteria included enrollment in Hospice, an intention to move out of the area in the next year, or for subjects without a related caregiver residing with them, the inability to provide informed consent. Individuals lacking capacity were enrolled if they had a related in-home caregiver to provide consent for their participation.

After baseline data were collected, subjects were randomly assigned to either an intervention or control group. Only data from the control group were included in these analyses. As part of the control condition, subjects were provided with an in-home assessment by an occupational therapist, who recommended environmental changes directed toward reducing falls, and advised participants to seek medical attention for their elevated risk of falling. One hundred seventy-five adults were randomized to the control group. Three had no follow-up data on falls, leaving 172 participants for inclusion in this analysis.

Baseline Data. A set of interview and questionnaire data were collected at baseline. These included demographic information, assessments of functional ability (activities of daily living (ADLs) [8] and instrumental ADLs [9]), the short form of the Geriatric Depression Scale [10] (GDS-SF), history of impaired vision or use of an ambulation assistive device, comorbid medical conditions [11], number of prescription medications, number of psychotropic medications (i.e., antidepressants, sedative hypnotics, antihistamines, antipsychotics), current exercise, and self-perception of health [12]. The MMSE was administered in a standardized manner by trained personnel. Subjects were first asked 10 orientation questions, followed by a simple 3-word auditory registration task. After a brief delay, typically lasting 1 to 2 minutes during which the subject was asked to spell a 5-letter word backwards, the participant was asked to recall the 3 words presented during the registration task. Remaining items assessed the ability to name 2 common objects, repeat a sentence, follow a 3-step command, read and follow a written instruction, write a sentence, and accurately copy overlapping pentagons. Points were tallied for a total possible score of 30 .

Prospective Recording of Falls. The rate of accidental falls over 1 year in the community was measured prospectively for all subjects. An accidental fall was defined as 'an event which results in a person coming to rest inadvertently on the ground or other lower level, and other than as a consequence of the following: sustaining a violent blow; loss of consciousness; sudden onset of paralysis, as in stroke; an epileptic seizure' [13]. To facilitate an accurate record of falls, participants kept daily diaries and mailed in monthly records of falls. Caregivers were asked to assist with falls recording. If a fall was reported on a study participant's monthly calendar, research personnel conducted a follow up telephone interview to confirm the occurrence of the fall, as well as details of the nature, cause and location of the reported event. At the end of the study, the rate of falls variable used for analyses was calculated by dividing number of days in the community into number of falls occurring while in the community $[7,14]$. Falls occurring on days spent in a hospital, communitybased residential facility, or nursing home were excluded from analyses.

\section{Statistical Methodology}

Numbers of falls in the community were modeled using a negative binomial (overdispersed Poisson) generalized additive regression model with a log link function. For each subject, (log) number of community days was included in the model as an off- 
Table 1. Participant characteristics

\begin{tabular}{|c|c|}
\hline Characteristics & $\begin{array}{l}\text { Subjects randomized to the } \\
\text { control condition }(n=172)\end{array}$ \\
\hline Age, years & $80.4 \pm 7.7$ \\
\hline Females, $\mathrm{n}$ & $134(77.9 \%)$ \\
\hline Education, years & $12.5 \pm 3.5$ \\
\hline Total MMSE score ${ }^{a}$ & $27.2 \pm 4.6$ \\
\hline Reported health status: $\mathrm{n}$ reporting overall health as fair or poor & $55(32.0 \%)$ \\
\hline Depression: $\mathrm{n}$ self-reporting diagnosis of depression & $58(33.7 \%)$ \\
\hline Arthritis: $\mathrm{n}$ self-reporting disease on list of comorbid conditions & $140(81.9 \%)$ \\
\hline Vision impairment: $\mathrm{n}$ unable to watch TV due to poor vision & $28(16.3 \%)$ \\
\hline Use of assistive device: $\mathrm{n}$ reporting use $>50 \%$ of time indoors & $60(34.9 \%)$ \\
\hline Exercise: $n$ reporting engaging in physical activity $\geq 4$ times/week & $27(20.2 \%)$ \\
\hline $\mathrm{n}$ reporting engaging in physical activity $1-3$ times/week & $51(38.1 \%)$ \\
\hline ADL scale: mean total score on Barthel Index ${ }^{b}$ & $88.7 \pm 16.5$ \\
\hline $\begin{array}{l}\text { IADL scale: mean number out of } 7 \text { IADLs that subjects report perfor } \\
\text { independently }{ }^{c}\end{array}$ & $4.9 \pm 2.2$ \\
\hline Alcohol use: $n$ reporting consumption of $\geq 3$ drinks/week & $15(8.7 \%)$ \\
\hline Caregiver in the home: $\mathrm{n}$ of participants living with someone & $79(45.9 \%)$ \\
\hline Hospitalizations: $\mathrm{n}$ of subjects hospitalized $\geq 1$ time in last 4 months & $30(17.4 \%)$ \\
\hline Stroke: $\mathrm{n}$ endorsing stroke on list of comorbid conditions & $40(23.3 \%)$ \\
\hline Falls in year prior to study entry: $\mathrm{n}$ reporting $\geq 2$ falls & $95(55.2 \%)$ \\
\hline$n$ reporting $\geq 1$ falls & $154(89.5 \%)$ \\
\hline Using psychotropic medication(s): $\mathrm{n}$ using $\geq 1$ specified medication ${ }^{\mathrm{d}}$ & $19(11.1 \%)$ \\
\hline
\end{tabular}

Values are means \pm SD or $\mathrm{n}$ with percentages in parentheses as indicated.

${ }^{a}$ Range of scores for MMSE: 0-30 points. Higher score indicates better global cognition.

${ }^{b}$ Subjects rated on level of independence on 10 items from the Barthel ADL scale: feeding, grooming, bathing, dressing, toileting, bowel and bladder control, transferring, walking and stairs. Range of scores: 0-100 points. Higher score indicates greater independence.

' Seven IADLs from the Lawton IADL scale including: housework, preparing meals, transportation, shopping, finances, managing medications, and telephoning. Range of scores: independent on 0-7 IADLs.

'Inquired about use of the following psychotropic medications: antidepressants, sedative hypnotics, antihistamines, antipsychotics.

set term to account for variable lengths of follow-up. MMSE score was included in the model as a penalized thin plate regression spline with smoothing parameter chosen by generalized crossvalidation [15]. All control subjects, regardless of MMSE score, were included in the analysis.

Models were fit both without covariates (univariate) and after covariate adjustment (multivariate). The multivariate model employed a stepwise algorithm using a 'change-in-estimate' method [16]. Variables resulting in the largest change in the average linear effect (average change in falls rate for a 1-point drop in MMSE score in the range 22-30) were added to the model as long as the addition of any of the remaining variables did not result in a $\geq 10 \%$ change in the average linear effect. Variables were considered for inclusion in the model in 2 stages. In the first stage, the following variables, identified as potential confounders, were evaluated for inclusion in the model: age, education, gender, depression (reported on a questionnaire of comorbid conditions) [11], vision impairment (self-report of being unable to watch television due to poor vision), alcohol use ( $>3$ days/week), history of stroke (as reported in questionnaire) [11], presence of a caregiver in the home, hospitalization in the last 4 months, fair to poor health status (as reported in questionnaire) [11], and use of one or more psychotropic medications. In the second stage, the following variables assessing mobility-related factors that may be associated with falls were considered for inclusion in the model: use of assistive device more than $50 \%$ of time indoors, arthritis (selfreport of disease on list of comorbid conditions), level of exercise (none, 1-3 times/week, or $\geq 4$ times/week), and ADLs (total score on Barthel Index [8]). A similar procedure was employed by van Schoor et al. [17], who referred to a similar set of variables as 'mediators' and entered them in their multivariate model, after first accounting for the contribution of confounders. Analyses were performed using R [18]. Age (years) and education (years of school) were modeled as penalized thin plate regression splines. All other variables were entered as dichotomous/categorical variables. 


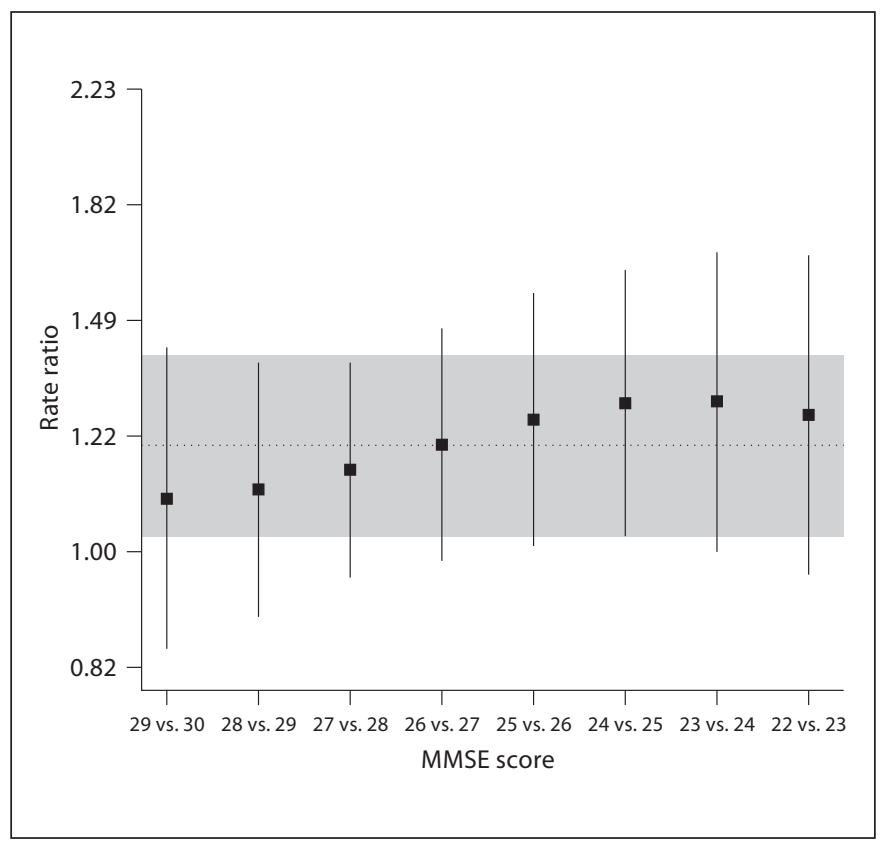

Fig. 1. Estimated rate ratios for falls and 95\% CIs for individual MMSE scores (relative to next highest MMSE score) down to an MMSE score of 22 based on multivariate negative binomial generalized additive regression model. (An MMSE score of 22 was the last score with more than 1 observation). In addition to MMSE score, the model includes the following covariates: score on an ADL checklist, current exercise, arthritis, and use of assistive device. Horizontal line and gray shaded region give point estimate and $95 \% \mathrm{CI}$ for average linear effect over this range of MMSE scores.

\section{Results}

Based on prospective tracking of falls, there were 315 falls in 162.82 person-years of follow-up in the 172 control group subjects with at least 1 follow-up day in the community. Table 1 presents the baseline characteristics, including MMSE score, for these 172 subjects. The overall falls rate for the control group was 1.93. Table 2 describes the number of subjects obtaining a given MMSE score, and the rate of falls per year in the community associated with each MMSE score.

Most participants (91\%) scored at or above a standard MMSE cutoff of $\geq 24 / 30$ for dementia. The mean MMSE score was 27.2 (SD 4.6). The mean age of study participants was 80.4 (SD 7.7) years; $78 \%$ of participants were women. The study population had a high level of morbidity: approximately $34 \%$ were depressed, $82 \%$ had arthritis, $16 \%$ had vision impairment, $35 \%$ used an assistive device and $17 \%$ were hospitalized in the 4 months prior to
Table 2. Number of subjects obtaining a given MMSE score and rate of falls per year in the community associated with individual MMSE score

\begin{tabular}{lrl}
\hline $\begin{array}{l}\text { MMSE } \\
\text { Score }\end{array}$ & $\mathrm{n}$ & $\begin{array}{l}\text { Average rate of falls per year } \\
\text { in the community }(95 \% \mathrm{CI})\end{array}$ \\
\hline 30 & 47 & $1.32(0.80,2.16)$ \\
29 & 26 & $1.59(1.10,2.30)$ \\
28 & 43 & $1.94(1.36,2.77)$ \\
27 & 24 & $2.41(1.60,3.65)$ \\
26 & 9 & $3.05(1.85,3.65)$ \\
25 & 7 & $3.92(2.14,7.17)$ \\
24 & 3 & $5.03(2.45,10.30)$ \\
23 & 3 & $6.37(2.77,14.63)$ \\
22 & 2 & $7.87(3.04,20.37)$ \\
\hline
\end{tabular}

the study. All participants were community-dwelling with many (54\%) living alone.

\section{Relationship between Decrements on MMSE and Rate of Falls}

Results from univariate and multivariate regression models describing the relationship between decrements on the MMSE and rate of falls are presented in table 3. In univariate analyses, lower MMSE scores in the range 2230 were associated with a log-linear increase in rate of falls (rate ratio 1.25, 95\% confidence interval (CI) 1.09$1.45, \mathrm{p}=0.0026)$. There was no evidence of non-linearity $(\mathrm{p}=0.99)$.

\section{Multivariate Model}

Using a stepwise, change-in-estimate method [16] to select covariates for inclusion in the multivariate model, scores on an index of ADL performance (Barthel), level of exercise, use of assistive device and arthritis were included in the multivariate (adjusted) model. After adjustment for these variables, unit decreases in MMSE scores in the range 22-30 remained associated with log-linear increases in the rate of falls (rate ratio 1.20 , 95\% CI 1.03$1.40, \mathrm{p}=0.021)$ (table 2). There was no evidence of nonlinearity $(\mathrm{p}=0.97)$.

Twelve subjects (7\%) had a self-reported diagnosis of dementia. Eight of these individuals scored below 24/30 on the MMSE. To assess how inclusion of individuals with more severe cognitive impairments may have influenced results, we calculated average linear effect estimates for incrementally narrower ranges of MMSE scores (e.g., MMSE 24-30, 26-30, and 28-30, instead of MMSE 22-30). The point estimates were largely unchanged in 
Table 3. Association of MMSE score with rate of falls in univariate and stepwise multivariate regression models

\begin{tabular}{|c|c|c|c|c|c|c|}
\hline & \multicolumn{3}{|c|}{ 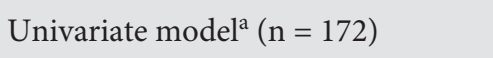 } & \multicolumn{3}{|c|}{ Multivariate (stepwise) model $^{\mathrm{a}, \mathrm{b}}(\mathrm{n}=171)^{\mathrm{c}}$} \\
\hline & Rate ratio & $95 \% \mathrm{CI}$ & $\mathrm{p}$ value & Rate ratio & $95 \%$ CI & $\mathrm{p}$ value \\
\hline MMSE score average linear effect $(22-30)^{\mathrm{d}}$ & 1.25 & $1.09,1.45$ & 0.0026 & 1.20 & $1.03,1.40$ & 0.0209 \\
\hline
\end{tabular}

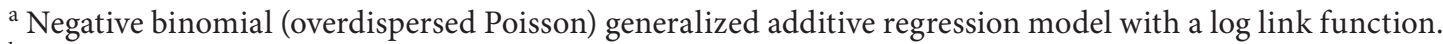

${ }^{\mathrm{b}}$ For multivariate model, variables resulting in the largest change in the average linear effect (average change in falls rate for a 1-point drop in MMSE score in the range 22-30) were added to the model until the addition of any of the remaining variables did not result in a $\geq 10 \%$ change in the average linear effect. Resulting model adjusted for Barthel score, assistive device use, current exercise and arthritis.

${ }^{c}$ Data on arthritis was not available for one subject.

${ }^{\mathrm{d}}$ Average rate ratio for a 1-point difference in MMSE scores in the range 22-30. (An MMSE score of 22 was the last score with more than 1 observation.)
}

the univariate analyses and slightly attenuated in the multivariate analysis, but there was no evidence that any of these estimates differed from each other. These results suggest a robust average linear effect across the range of observed MMSE scores.

Figure 1 presents the estimated rate ratios for the comparison of a given MMSE score with the next highest MMSE score. For example, the rate of falls associated with a MMSE score of 25 was significantly higher than the rate of falls associated with a score of 26 (rate ratio $1.26,95 \%$ CI 1.02-1.56). As noted previously, these estimates are all consistent with a common effect of a single point loss of MMSE score over this range of MMSE scores (overall rate ratio 1.20, 95\% CI 1.03-1.40).

\section{Discussion}

These analyses from the Kenosha County Falls Prevention Study [7] suggest that in patients at risk for falling, unit decrements on a widely-used global cognitive scale, the MMSE, are incrementally associated with risk for falling, even in the upper range of scores. In our sample, all subjects were at risk for falls, and many exhibited a high rate of comorbidity. Of note, most participants in this community-dwelling sample had an MMSE score above a cutoff associated with dementia (i.e. median MMSE $=28 / 30$ ). The association between MMSE and falls persisted across the range of scores from 22 to 29 , and remained after adjusting for covariates. Several studies have reported an increased risk for falls in patients who carry the diagnosis of dementia (e.g., $[1,19,20])$ emphasizing the relationship between dementia and falls [3]
Other studies have found an increased risk for falls with MMSE scores below 24 [3-5]. Our data suggest that for older adults who have a history of falls, elevations in risk for future falls occur with even very mild cognitive deficits.

In contrast, another population-based study found that the relationship between point loss on the MMSE and rate of falls was no longer significant after adjusting for age [17]. This finding may have resulted from distinct differences between subject groups. Participants in our study were all at high risk for falls as part of the eligibility criteria for the study. In addition, participants all had sought recruitment into an intervention study to reduce falls. Our subjects were older on average, and had a high level of disease comorbidity. For example, $82 \%$ of our participants had arthritis, and over $20 \%$ had sustained a stroke, compared to only $10 \%$ of participants with stroke in the population-based study. It may be that the relationship between rate of falls and cognitive decline becomes stronger as patients accrue multiple risk factors for falls. Of note, our subjects, who were enrolled in the study based upon their risk for falls may be more similar to patients found in specialty clinics (e.g. falls, dementia, neurology, rehabilitation clinics, etc.), compared to subjects enrolled in a population-based study. Understanding that there may be an increase in falls risk associated with subtle cognitive changes, clinicians may improve their ability to identify their patients' risk of future falls and institute appropriate prevention strategies. Specifically our data suggest that, for those with a history of falls, an MMSE score $<30$ should be considered as an important indicator of fall risk independent of other risk factors, with risk increasing with each unit decrement on MMSE, 
down to an MMSE score of 22. Given the small number of subjects with MMSE scores below 22, we are unable to determine if falls risk continues to increase incrementally with additional unit point declines for MMSE scores below 22 .

The neurobiological basis for the association between falling and subtle cognitive deficits needs clarification. It has been demonstrated that impairments in judgment, attention, or executive function may predispose older adults to perform unsafe tasks or to execute them in a perilous manner [21]. As such, it is possible that the association between falls risk and mild decrements on the MMSE is due directly to the effect of deficits in key cognitive domains, such as executive function. For example, recent work shows that impairment in executive function is associated with decreased ability to modulate gait in the setting of a dual task (for example walking and performing mental arithmetic), and that this is particularly true for older adults prone to falls [22-24]. We were not able to characterize the nature of cognitive deficits in this group, and cannot determine if deficits in particular cognitive domains, such as executive function or memory, were uniquely associated with elevations in falls rate. This is an important area for future study.

This study has limitations. First, the small sample size for MMSE scores below 22 precluded meaningful analysis of the association between MMSE score and rate of falls for those scores. Second, there was a relatively small number of subjects with MMSE scores in the range from 22 to 26; despite the small sample size, there was a significant association between MMSE score and rate of falls for MMSE scores from 22 to 26. Regardless, further evaluation with larger sample sizes and using a more detailed array of cognitive tests would be important next steps to corroborate these findings. Third, a limitation of our analyses is that our data apply to an at-risk cohort, residing in one geographic area, who self-selected to enroll in an intervention study. The same conclusions may not apply to older adults without a recent history of falls, or those disinclined or unable to seek intervention. Strengths of these analyses include the systematic, monthly tracking of falls prospectively and the derivation of community-based falls rate, adjusting for number of days subjects spent in the community.

In summary, findings from these analyses expand our understanding of the relationship between cognitive decline and risk for falls. Among older adults with a history of falls, estimations of future falls risk can be improved by using data from a simple screening tool, the MMSE, which is often incorporated in a routine primary care assessment. Specifically, there is an increased falls risk for each unit decrement from a score of 30 on the MMSE. For clinicians caring for the expanding population of older adults, a fuller awareness of the association between subtle cognitive impairment and risk for falls may lead to better identification of the degree of risk among at-risk patients. In addition, knowledge of this relationship may provide the impetus to develop interventions targeting subtlety impaired patients and their caregivers.

\section{Acknowledgments}

This work was supported by NIH-NIA grant K23 AG024302 (PI: C.E.G.), the Kenosha County Multi-Factorial Falls Prevention Program for Older Adults; A Wisconsin Family Care Aging and/or Disability Resource Center Prevention Funding Grant (PI: J.E.M.); and the Geriatric Research, Education and Clinical Center and Mental Health Service of the Wm. S. Middleton Memorial VA, Madison, Wisc. This is GRECC Manuscript Number: 2007-015.

\section{References}

1 Horikawa E, Matsui T, Arai H, Seki T, Iwasaki K, Sasaki H: Risk of falls in Alzheimer's disease: a prospective study. Intern Med 2005;44:717-721.

-2 Folstein MF, Folstein SE, McHugh PR: 'Minimental state'. A practical method for grading the cognitive state of patients for the clinician. J Psychiatr Res 1975;12:189-198.

-3 Anstey KJ, von Sanden C, Luszcz MA: An 8year prospective study of the relationship between cognitive performance and falling in very old adults. J Am Geriatr Soc 2006;54: 1169-1176.
4 Tinetti ME, Speechley M, Ginter SF: Risk factors for falls among elderly persons living in the community. N Engl J Med 1988;319: 1701-1707.

5 Graafmans WC, Ooms ME, Hofstee HM, Bezemer PD, Bouter LM, Lips P: Falls in the elderly: a prospective study of risk factors and risk profiles. Am J Epidemiol 1996;143: 1129-1136.

-6 Harvan JR, Cotter V: An evaluation of dementia screening in the primary care setting. J Am Acad Nurse Pract 2006;18:351-360.
7 Mahoney JE, Shea TA, Przybelski R, Jaros L, Gangnon R, Cech S, Schwalbe A: Kenosha county falls prevention study: a randomized, controlled trial of an intermediate-intensity, community-based multifactorial falls intervention. J Am Geriatr Soc 2007;55:489-498.

-8 Mahoney FI, Barthel DW: Functional evaluation: the Barthel Index. Md State Med J 1965;14:61-65.

9 Lawton MP, Brody EM: Assessment of older people: self-maintaining and instrumental activities of daily living. Gerontologist 1969; 9:179-186. 
10 Sheikh J, Yesavage J: Geriatric depression scale (GDS): recent evidence and development of a shorter version; in Brink TL (ed): Clinical Gerontology: A Guide to Assessment and Intervention. New York, Haworth Press, 1986, pp 165-173.

-11 Stuck AE, Aronow HU, Steiner A, Alessi CA, Büla CJ, Gold MN, Yuhas KE, Nisenbaum R, Rubenstein LZ, Beck JC: A trial of annual inhome comprehensive geriatric assessments for elderly people living in the community. $\mathrm{N}$ Engl J Med 1995;333:1184-1189.

12 Ware JE Jr, Sherbourne CD: The MOS 36item short-form health survey (SF-36). I. Conceptual framework and item selection. Med Care 1992;30:473-483.

13 The prevention of falls in later life. A report of the Kellogg international work group on the prevention of falls by the elderly. Dan Med Bull 1987;34(suppl 4):1-24.
14 Mahoney JE, Palta M, Johnson J, Jalaluddin M, Gray S, Park S, Sager M: Temporal association between hospitalization and rate of falls after discharge. Arch Intern Med 2000; 160:2788-2795.

15 Wood SN: Generalized Additive Models: An Introduction with R. Boca Raton, FL, Chapman \& Hall/CRC, 2006.

16 Greenland S: Modeling and variable selection in epidemiologic analysis. Am J Public Health 1989;79:340-349.

17 van Schoor NM, Smit JH, Pluijm SM, Jonker C, Lips P: Different cognitive functions in relation to falls among older persons. Immediate memory as an independent risk factor for falls. J Clin Epidemiol 2002;55:855-862.

18 Team RDC: R: A language and environment for statistical computing. Vienna, Austria, $\mathrm{R}$ Foundation for Statistical Computing, 2005.

19 Buchner DM, Larson EB: Falls and fractures in patients with Alzheimer-type dementia. JAMA 1987;257:1492-1495.
20 van Dijk PT, Meulenberg OG, van de Sande HJ, Habbema JD: Falls in dementia patients. Gerontologist 1993;33:200-204.

21 Chapman GJ, Hollands MA: Evidence that older adult fallers prioritise the planning of future stepping actions over the accurate execution of ongoing steps during complex locomotor tasks. Gait Posture 2007;26:59-67.

22 Allali G, Kressig RW, Assal F, Herrmann FR, Dubost V, Beauchet O: Changes in gait while backward counting in demented older adults with frontal lobe dysfunction. Gait Posture 2007;26:572-576

23 Holtzer R, Verghese J, Xue X, Lipton RB: Cognitive processes related to gait velocity: Results from the Einstein aging study. Neuropsychology 2006;20:215-223.

-24 Springer S, Giladi N, Peretz C, Yogev G, Simon ES, Hausdorff JM: Dual-tasking effects on gait variability: the role of aging, falls, and executive function. Mov Disord 2006;21: 950-957. 